\title{
Comparison between muscle-preserving selective laminectomy and laminoplasty for multilevel cervical spondylotic myelopathy
}

\author{
Satoshi Nori ${ }^{1}$, Tateru Shiraishi ${ }^{2}$, Ryoma Aoyama $^{1}$ \\ ${ }^{1}$ Department of Orthopedic Surgery, Tokyo Dental College Ichikawa General Hospital, Chiba, Japan; ${ }^{2}$ Shiraishi Spine Clinic, Tokyo, Japan \\ Correspondence to: Satoshi Nori, MD, PhD. Tokyo Dental College Ichikawa General Hospital, 5-11-13 Sugano, Ichikawa, Chiba 272-8513, Japan. \\ Email: satoshi_nori@2003.jukuin.keio.ac.jp. \\ Provenance and Peer Review: This article was commissioned by the Editorial Office, Annals of Translational Medicine. The article did not undergo \\ external peer review. \\ Comment on: Yu Z, He D, Xiong J, et al. Extensor muscle-preserving laminectomy in treating multilevel cervical spondylotic myelopathy compared \\ with laminoplasty. Ann Transl Med 2019;7:472.
}

Submitted Nov 11, 2019. Accepted for publication Nov 22, 2019.

doi: $10.21037 /$ atm.2019.11.132

View this article at: http://dx.doi.org/10.21037/atm.2019.11.132

\section{Introduction}

The recently reported study conducted by Yu et al. compared the clinical and radiologic outcomes of skip laminectomy, one of the surgical techniques included in muscle-preserving selective laminectomy (SL) and expansive open-door laminoplasty (ELAP) performed for the treatment of multilevel cervical spondylotic myelopathy (CSM) (1). This prospective study demonstrated the advantages of skip laminectomy in reducing intraoperative blood loss, hospital stay, postoperative visual analogue scale (VAS) score, and axial symptoms, along with preserving the postoperative cervical range of motion (ROM). The authors concluded that skip laminectomy is an effective, safe, and minimally invasive method as it preserves the posterior cervical structure. We believe that this study informs surgeons about an alternative to laminoplasty for the treatment for CSM.

Until the 1960s, posterior cervical decompression had been performed only by laminectomy. However, surgeons recognized various complications of laminectomy, such as kyphotic deformity, segmental instability, and late neurologic deterioration $(2,3)$. After the advent of highspeed surgical drills in the 1970s, Japanese surgeons first performed and developed the laminoplasty procedure, attempting to avoid these complications (4-6). Currently, ELAP or double-door laminoplasty (DDLP) is widely used as a multiple posterior decompression technique for CSM. However, the superiority of cervical laminoplasty as compared to conventional laminectomy remains uncertain because the former sometimes results in poor surgical outcomes due to complications, such as re-closure of the expanded laminae, dislodgement of the expanded laminae, and unintentional bony fusion between the preserved laminae. In addition, laminoplasty failed to solve the postoperative problem of axial symptoms and development of cervical malalignment. Under such circumstances, in 1998, Shiraishi developed a less invasive technique to expose the cervical spine laminae, which preserves the extensor mechanism of the cervical spine $(7,8)$. SL is performed by applying Shiraishi's technique to posterior cervical decompression.

We currently limit the surgical indications for SL to (I) a local kyphosis of $<20^{\circ}$; (II) spondylolisthesis of $<3.5 \mathrm{~mm}$; (III) an ossification of the posterior longitudinal ligament (OPLL) occupancy ratio of $<60 \%$. The levels of decompression are identified by complete obstruction of the subarachnoid space on preoperative dynamic cervical myelography-computed tomography (CM-CT) or dynamic T2-weighted magnetic resonance images (MRI) with the neck in a neutral or extended position (9). In our previous series, the mean laminectomy width was $15-19 \mathrm{~mm}$ (10), but we emphasize that on the preoperative axial images of these scans, the laminectomy width is kept $2-3 \mathrm{~mm}$ wider than the spinal cord (9). This is key for success in SL; to achieve simultaneous secure decompression of the spinal cord and minimum invasion of the stabilizing structures. Two consecutive and adjacent intervertebral levels are decompressed by mono-laminectomy, which removes a single lamina, the upper half of the lower adjacent lamina, 

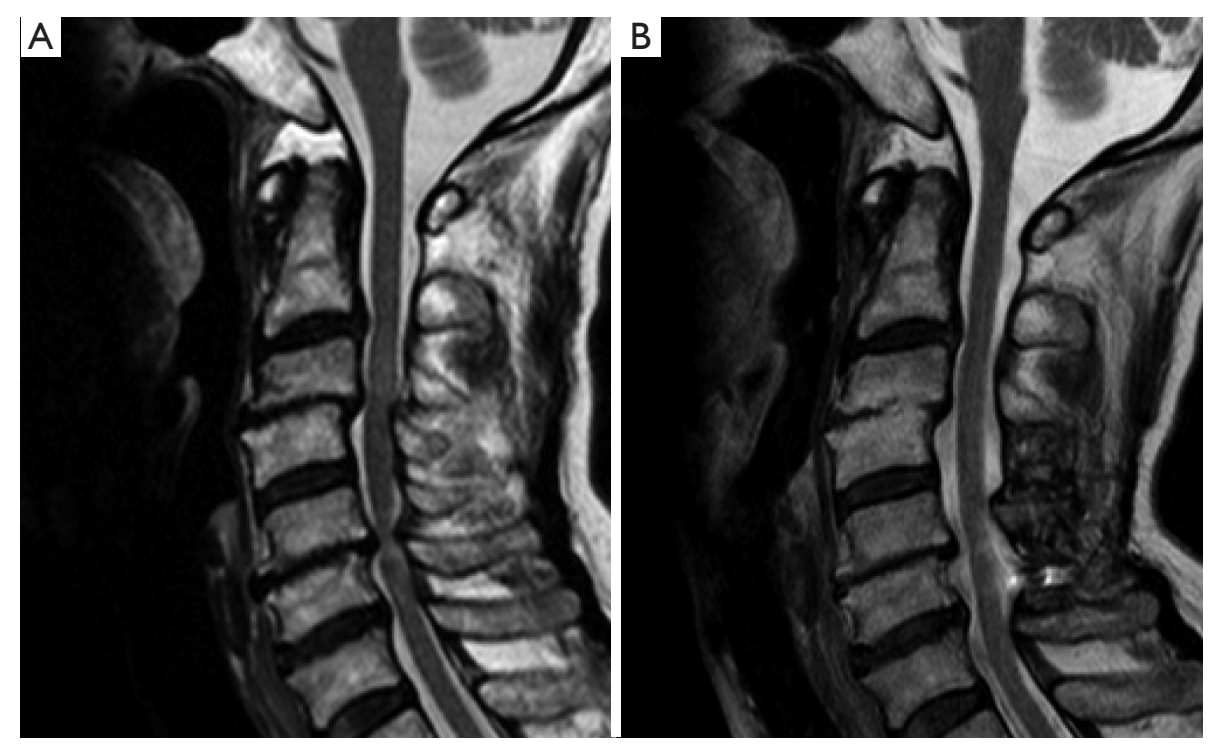

Figure 1 A 66-year-old woman underwent C4-C6 skip laminectomy. (A) Preoperative T2-weighted MRI; (B) a T2-weighted MRI three years after surgery presented successful decompression. MRI, magnetic resonance image.
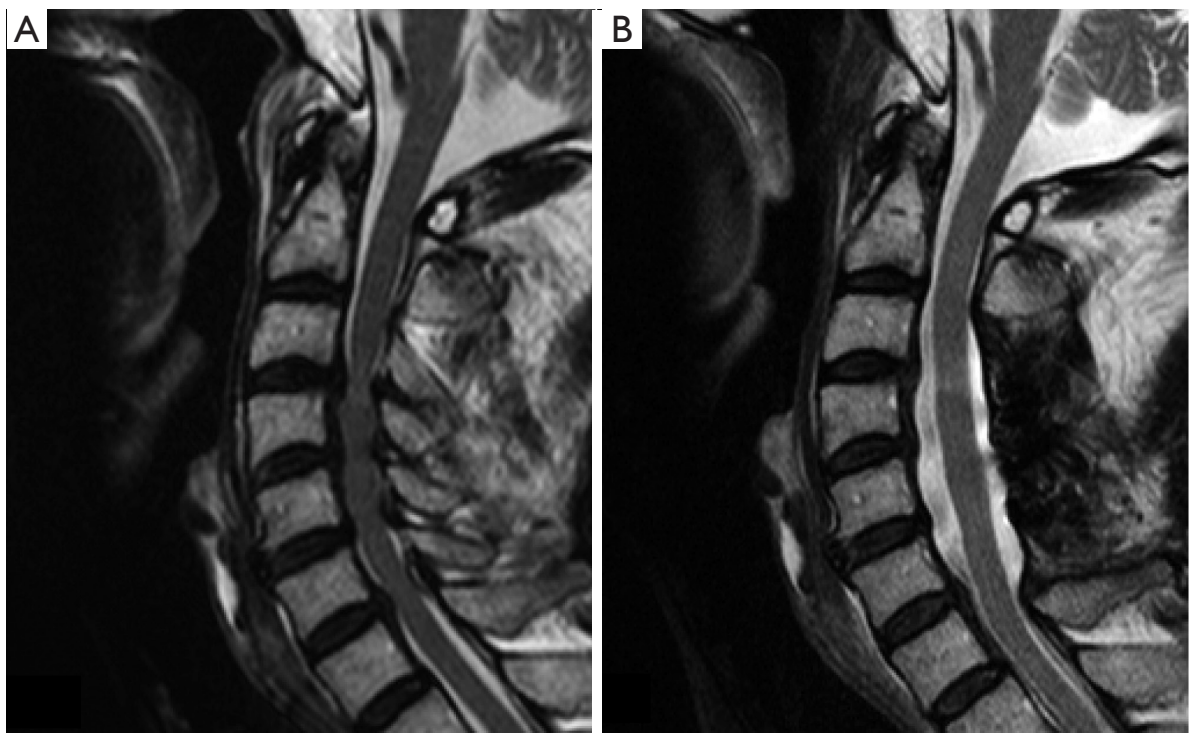

Figure 2 A 64-year-old man underwent C3-C6 selective laminectomy. (A) Preoperative T2-weighted MRI; (B) a T2-weighted MRI two years after surgery presented successful decompression. MRI, magnetic resonance image.

and the flavum at the affected levels (7). Decompression of three consecutive adjacent intervertebral levels is accomplished by double laminectomy of the adjacent two laminae between the affected levels (11). For the CSM patients with spinal cord compression of more than four levels, skip laminectomy is indicated if it is possible to leave at least one lamina unremoved between the affected levels.
In such cases, it goes without saying that preoperative imaging studies show no spinal cord compression by the lamina (or laminae) to be preserved but the compression only at the upper and lower adjacent levels of each one of those laminae (Figure 1). For OPLL or for developmental canal stenosis, consecutive laminectomy of four or more laminae is usually performed (Figure 2) (11). For the 
patients with involvement of $\mathrm{C} 2$, we used a technique for muscle-preserving DDLP for the axis (11).

\section{Reviewer's comments}

We followed the results of each evaluation indicator presented by the authors and made comments on: (I) functional outcomes; (II) radiological analyses; (III) postoperative cervical pain; (IV) intraoperative bleeding; (V) C5 palsy; and (VI) limitations of the study.

\section{Functional outcomes}

The study prospectively recruited 20 patients with multilevel CSM who underwent skip laminectomy and 24 patients with multilevel CSM who received ELAP. After surgery, the postoperative JOA score showed improvements in both groups, with no significant difference in the recovery rate of the JOA score in either group. These results are consistent with a previous prospective randomized study comparing skip laminectomy and DDLP (12). Skip laminectomy showed good functional recovery in multilevel CSM patients as compared to laminoplasty.

\section{Radiological analyses}

\section{Cervical sagittal alignment}

The authors concluded that postoperative cervical curvature index had not significantly changed in both groups. On the contrary, our previous study showed that postoperative curvature in the ELAP group was significantly smaller than that of the preoperative group, while there was no significant difference regarding skip laminectomy (13). In terms of preoperative cervical alignment, the inclusion criteria of this study were a local kyphosis of $<10^{\circ}$, while our indication for SL was a local kyphosis of $<20^{\circ}$. The study could have shown a different result in the postoperative alignment of each group if the authors' criteria was the same as ours.

Damage to the stabilizing structures of the cervical spine also negatively influences the cervical alignment. SL can be securely performed by strictly limiting the lateral exposure inside the medial border of the bilateral facet joints. However, satisfactory posterior decompression cannot be accomplished by laminoplasty without creating lateral gutters away from the edges of the spinal cord. Thus, the lateral exposure is always kept wider in laminoplasty as compared to SL, damaging the facet joint, its capsule, and the origins of the multifidus. Laminoplasty also compromises the deep extensor muscles (DEMs) that act as dynamic stabilizers of the cervical spine, such as the semispinalis cervicis and multifidus, by removing them from the spinous processes. Atrophy of the DEMs is a significant risk factor for postoperative development of poor cervical alignment (14). We previously reported that postoperative atrophy of the DEMs was significantly greater in ELAP than in skip laminectomy (13). It is speculated that damage to the posterior cervical structures by laminoplasty causes postoperative cervical malalignment.

Cervical sagittal vertical axis (SVA) is also an important indicator to evaluate the cervical alignment. Our previous study demonstrated that SL performed to decompress laminae at the C6 single level and C5-C6 consecutive two levels left the C2-C7 angle unaffected without increasing C2-C7 SVA after surgery (15). Decompression at three consecutive levels C4-C6 and four consecutive levels C3C6 presented a slight increase in C2-C7 SVA, while the C2-C7 angle increased as a compensation for the SVA increase (15). Since the authors in the study analyzed the cervical curvature index alone, it is difficult to evaluate the difference created in the C2-C7 angle and the C2-C7 SVA between skip laminectomy and ELAP. We speculate that the intensity of surgical invasion on the posterior structures is not significantly different between skip laminectomy and C5-6 SL. Therefore, it is understood that the study results demonstrated no change in the cervical curvature index for the skip group. Previous studies showed that the C2C7 angle decreased despite the increase in C2-C7 SVA after DDLP or ELAP $(16,17)$. The study would be more effective if the authors conducted a more detailed analysis of cervical sagittal alignment and measured the cervical SVA.

\section{Cervical ROM}

The study results demonstrated that cervical ROM was not significantly changed after skip laminectomy, while it was significantly decreased after ELAP. A previous prospective nonrandomized study and retrospective study demonstrated that cervical ROM after skip laminectomy was successfully preserved as compared to laminoplasty $(13,18)$. Another previous study showed that cervical ROM slightly diminished after SL for C4-C6 consecutive three-level and C3-C6 consecutive four-level decompression, however, ROM did not diminish after SL for C6 single-level and C5C6 consecutive two-level decompression (15). Since the preservation of posterior structures by skip laminectomy is almost no different from that of SL at C5-C6, it is reasonable that the study demonstrated no reduction of cervical ROM after skip laminectomy. Laminoplasty induces damage to the facet joints, leading to restriction of cervical $\mathrm{ROM}$ as a result of unexpected bony fusion between the facet joints or preserved laminae. Since lateral exposure is 
strictly limited in SL without exposing the bilateral facet joints during the surgery, we did not observe such bony fusion in our SL patients. Unintentional or unexpected bony fusion by laminoplasty inevitably causes a reduction of cervical ROM.

\section{Postoperative cervical pain}

The study showed lower VAS and less axial symptoms in the skip group as compared to the ELAP group. This is consistent with a previous prospective nonrandomized study comparing skip laminectomy with DDLP (18). We suggested that lesser damage to the facet joints and DEMs by skip laminectomy resulted in less cervical pain and axial symptoms. The study also showed that hospital stay was shorter in skip group as compared to ELAP group. This is because skip laminectomy is less invasive to posterior stabilizing structures than ELAP, leading to faster pain relief. The authors of the study recommended a soft collar for 1 month after the surgery; however, we did not use it since we performed SL first. Since then, the postoperative period for our patients has been uneventful.

\section{Intraoperative bleeding}

The authors concluded that the intraoperative blood loss was lesser in the skip group (average: $73.6 \mathrm{~g}$ ) as compared to the ELAP group (average: $239.8 \mathrm{~g}$ ). It is consistent with the above-mentioned prospective nonrandomized study comparing skip laminectomy (average blood loss: $50 \mathrm{~mL}$ ) with DDLP (average blood loss: $105 \mathrm{~mL}$ ) (18). We observed that limited lateral exposure and preservation of DEMs in the skip group significantly reduced the blood loss at surgery. The mean blood loss in our previous SL series was around $10 \mathrm{~g}(9,15)$, which was lesser than other studies. This discrepancy might be related to the exposure technique or the decompression procedure. We used an operating microscope throughout the surgery to keep the field dry and cause minimum damage to the extensor muscles and facet joints. Removing bone using a high-speed drill with a diamond-tipped burr and not a cutting burr is another method to reduce intraoperative bleeding.

\section{C5 palsy}

The study presented that the incidences of C5 palsy were $0 \%$ after skip laminectomy and $16.7 \%$ after ELAP. Several authors reported that wide posterior decompression facilitated the backward shift of the spinal cord with the excessively protracted nerve root, consequently raising the risk of C5 palsy (19-21). We observed that the occurrence of palsy was affected by the width of laminectomy in SL. Our previous study demonstrated that SL performed with a laminectomy of up to $2-3 \mathrm{~mm}$ wider than the spinal cord significantly reduced the incidence of C5 palsy $(1.2 \%)$ as compared to SL with laminectomy being wider (9.2\%) (22). The authors performed skip laminectomy with a width of approximately $15 \mathrm{~mm}$, and it was consistent with our narrow SL with its average width of $15-19 \mathrm{~mm}$ (10). Lower incidence of C5 palsy in the skip group may be related to the narrow removal of the affected laminae to prevent excessive posterior spinal cord shift to some extent (22).

Direct or thermal injury to the C5 nerve root during the gutter-making process using a high-speed drill is another cause of palsy $(23,24)$. To achieve adequate decompression of the spinal cord, laminoplasty needs to make lateral gutters sufficiently away from the bilateral spinal cord edges. However, the affected spinal cords in CSM do not always run straight in the spinal canal and sometimes take a tortuous course due to anterior compression factors pushing the cord to one side. According to the location of the spinal cord, we adjusted the position of the laminectomy a few millimeters to the affected side, with its width kept as planned to securely decompress the spinal cord (22). Thus, narrow SL can prevent direct injury to the C5 nerve root and reduce the incidence of palsy.

\section{Limitations of the study}

There are some limitations to the study. First, it was a single-institution study and had a relatively limited number of patients with a short follow-up period. Second, the levels of decompression in both groups were not presented in the study, which intensified heterogeneity. Third, the study included patients with previous cervical spine surgeries and those with foraminotomy combined with SL. The previous or combined surgeries could have affected the results. Damage to the facet joints and their capsules by foraminotomy can negatively impact the postoperative cervical alignment. It would have proved more valuable if the authors had analyzed the postoperative atrophy of the DEMs.

\section{Discussion}

Here, we will discuss the cost effectiveness of SL that was not addressed by the authors. Previous papers have reported problems related to preserved laminae, such as iatrogenic stenosis due to re-closure of the expanded 
laminae and injury to the spinal cord by the dislodged laminar door. To prevent these, surgeons have frequently performed additional instrumentation to firmly hold the expanded laminae in place. For example, the affected laminae in ELAP are fixed with threads attached to anchor screws or mini-plates and screws, while those in DDLP are attached with mini-plates and screws or hydroxyapatite spacers. Conventional wide cervical laminectomy is also usually combined with multiple instrumentation and fixation to prevent postoperative development of kyphosis or instability. In these situations, the cost for medical care increases exponentially and burdens countries' economies. Moreover, instrument fixation has potential risks, such as infection, spinal cord injury caused by the dislodged implants, and vascular or nerve injury due to misplaced screws. Those complications certainly cause prolonged hospital stays with complicated postoperative care and a delayed return to work. A recent meta-analysis comparing mini-plate fixation versus suture suspensory fixation in ELAP showed that mini-plate fixation was associated with greater surgical trauma (i.e., additional operation time and blood loss) (25). In most SL cases, successful spinal cord decompression can be achieved by maintaining cervical curvature, thereby eliminating the necessity of additional fixation using expensive internal devices. Postoperative care in SL is simplified with short hospital stays and early returns to work because it is minimally invasive to the posterior stabilizing structures of the neck. Therefore, we conclude that SL is a safe, secure, and cost-effective procedure. The study conducted by Yu et al. provides beneficial information that enables surgeons to choose a proper surgical method to treat cervical myelopathy. In the future, a randomized controlled study with a large patient population and longterm follow-up remains expected to confirm the results of past research.

\section{Acknowledgments}

Funding: None.

\section{Footnote}

Conflicts of Interest: The authors have no conflicts of interest to declare.

Ethical Statement: The authors are accountable for all aspects of the work in ensuring that questions related to the accuracy or integrity of any part of the work are appropriately investigated and resolved.
Open Access Statement: This is an Open Access article distributed in accordance with the Creative Commons Attribution-NonCommercial-NoDerivs 4.0 International License (CC BY-NC-ND 4.0), which permits the noncommercial replication and distribution of the article with the strict proviso that no changes or edits are made and the original work is properly cited (including links to both the formal publication through the relevant DOI and the license). See: https://creativecommons.org/licenses/by-nc-nd/4.0/.

\section{References}

1. Yu Z, He D, Xiong J, et al. Extensor muscle-preserving laminectomy in treating multilevel cervical spondylotic myelopathy compared with laminoplasty. Ann Transl Med 2019;7:472.

2. Guigui P, Benoist M, Deburge A. Spinal deformity and instability after multilevel cervical laminectomy for spondylotic myelopathy. Spine (Phila Pa 1976) 1998;23:440-7.

3. Albert TJ, Vacarro A. Postlaminectomy kyphosis. Spine (Phila Pa 1976) 1998;23:2738-45.

4. Kawai S, Sunago K, Doi K, et al. Cervical laminoplasty (Hattori's method). Procedure and follow-up results. Spine (Phila Pa 1976) 1988;13:1245-50.

5. Satomi K, Nishu Y, Kohno T, et al. Long-term followup studies of open-door expansive laminoplasty for cervical stenotic myelopathy. Spine (Phila Pa 1976) 1994;19:507-10.

6. Seichi A, Takeshita K, Ohishi I, et al. Long-term results of double-door laminoplasty for cervical stenotic myelopathy. Spine (Phila Pa 1976) 2001;26:479-87.

7. Shiraishi T, Kato M, Yato Y, et al. New techniques for exposure of posterior cervical spine through intermuscular planes and their surgical application. Spine (Phila Pa 1976) 2012;37:E286-96.

8. Shiraishi T. Skip laminectomy--a new treatment for cervical spondylotic myelopathy, preserving bilateral muscular attachments to the spinous processes: a preliminary report. Spine J 2002;2:108-15.

9. Nori S, Shiraishi T, Aoyama R, et al. Extremely high preoperative C7 slope limits compensatory cervical lordosis after muscle-preserving selective laminectomy. Eur Spine J 2018;27:2029-37.

10. Nori S, Shiraishi T, Aoyama R, et al. Narrow width of muscle-preserving selective laminectomy demonstrated sufficient surgical outcomes and reduced surgical invasiveness. J Clin Neurosci 2018;52:60-5. 
11. Shiraishi T, Aoyama R. Selective Cervical Laminectomy of Limited Width by Muscle-Sparing Technique Using Anatomical Plane Exposures. In: Koller H, Robinson Y. editors. Cervical Spine Surgery: Standard and Advanced Techniques. Springer Nature, 2019:437-41.

12. Yukawa Y, Kato F, Ito K, et al. Laminoplasty and skip laminectomy for cervical compressive myelopathy: range of motion, postoperative neck pain, and surgical outcomes in a randomized prospective study. Spine (Phila $\mathrm{Pa}$ 1976) 2007;32:1980-5.

13. Shiraishi T, Fukuda K, Yato Y, et al. Results of skip laminectomy-minimum 2-year follow-up study compared with open-door laminoplasty. Spine (Phila Pa 1976) 2003;28:2667-72.

14. Nori S, Iwanami A, Yasuda A, et al. Risk factor analysis of kyphotic malalignment after cervical intramedullary tumor resection in adults. J Neurosurg Spine 2017;27:518-27.

15. Nori S, Shiraishi T, Aoyama R, et al. Muscle-Preserving Selective Laminectomy Maintained the Compensatory Mechanism of Cervical Lordosis After Surgery. Spine (Phila Pa 1976) 2018;43:542-9.

16. Sakai K, Yoshii T, Hirai T, et al. Impact of the surgical treatment for degenerative cervical myelopathy on the preoperative cervical sagittal balance: a review of prospective comparative cohort between anterior decompression with fusion and laminoplasty. Eur Spine J 2017;26:104-12.

17. Lee CH, Jahng TA, Hyun SJ, et al. Expansive Laminoplasty Versus Laminectomy Alone Versus Laminectomy and Fusion for Cervical Ossification of the Posterior Longitudinal Ligament: Is There a Difference in the Clinical Outcome and Sagittal Alignment? Clin Spine Surg 2016;29:E9-15.

18. Sivaraman A, Bhadra AK, Altaf F, et al. Skip laminectomy and laminoplasty for cervical spondylotic myelopathy: a prospective study of clinical and radiologic outcomes. J Spinal Disord Tech 2010;23:96-100.

19. Tsuzuki N, Zhogshi L, Abe R, et al. Paralysis of the arm after posterior decompression of the cervical spinal cord. I. Anatomical investigation of the mechanism of paralysis. Eur Spine J 1993;2:191-6.

20. Radcliff KE, Limthongkul W, Kepler CK, et al. Cervical laminectomy width and spinal cord drift are risk factors for postoperative C5 palsy. J Spinal Disord Tech 2014;27:86-92.

21. Shiozaki T, Otsuka H, Nakata Y, et al. Spinal cord shift on magnetic resonance imaging at 24 hours after cervical laminoplasty. Spine (Phila Pa 1976) 2009;34:274-9.

22. Nori S, Aoyama R, Ninomiya K, et al. Cervical laminectomy of limited width prevents postoperative C5 palsy: a multivariate analysis of 263 musclepreserving posterior decompression cases. Eur Spine J 2017;26:2393-403.

23. Hosono N, Miwa T, Mukai Y, et al. Potential risk of thermal damage to cervical nerve roots by a high-speed drill. J Bone Joint Surg Br 2009;91:1541-4.

24. Takenaka S, Hosono N, Mukai Y, et al. The use of cooled saline during bone drilling to reduce the incidence of upper-limb palsy after cervical laminoplasty: clinical article. J Neurosurg Spine 2013;19:420-7.

25. Liu FY, Ma L, Huo LS, et al. Mini-plate fixation versus suture suspensory fixation in cervical laminoplasty: A meta-analysis. Medicine (Baltimore) 2017;96:e6026.
Cite this article as: Nori S, Shiraishi T, Aoyama R. Comparison between muscle-preserving selective laminectomy and laminoplasty for multilevel cervical spondylotic myelopathy. Ann Transl Med 2020;8(5):160. doi: 10.21037/atm.2019.11.132 